\title{
Effects of functionalization and side defects on single-photon emission in boron nitride quantum dots
}

\author{
Farzaneh Shayeganfar, ${ }^{1,6, *}$ M. Reza Rahimi Tabar, ${ }^{2}$ A. Simchi, ${ }^{3,4}$ and Javad Beheshtian ${ }^{5,6, \dagger}$ \\ ${ }^{1}$ Department of Civil and Environmental Engineering, Rice University, Houston, Texas 77005, USA \\ ${ }^{2}$ Department of Physics, Sharif University of Technology, 14588 Tehran, Iran \\ ${ }^{3}$ Department of Material Science and Engineering, Sharif University of Technology, 11365-9466 Tehran, Iran \\ ${ }^{4}$ Institute for Nanoscience and Nanotechnology, Sharif University of Technology, 14588 Tehran, Iran \\ ${ }^{5}$ Department of Physics, University of Antwerp, Groenenborgerlaan 171, B-2020 Antwerpen, Belgium \\ ${ }^{6}$ Department of Chemistry, Shahid Rajaee Teacher Training University, 16875-163 Tehran, Iran \\ (Received 12 April 2017; revised manuscript received 20 September 2017; published 23 October 2017)
}

\begin{abstract}
Boron nitride quantum dots (BNQDs) functionalized with chemical ligands exhibit intriguing optoelectronic properties due to the quantum confinement effect. This paper presents peculiar insights on the effect of side defects on the electronic structure and optical properties of BNQDs functionalized with different chemical bonds including hydrogen $(\mathrm{H})$, nitrogen $(\mathrm{N})$, hydroxyl $(\mathrm{OH})$, amine $\left(\mathrm{NH}_{2}\right)$, and thiol groups (inspired by experimental reports of functionalized BN nanosheets and nanotubes) Weng et al., Chem. Soc. Rev. 45, 3989 (2016). Hybrid density functional simulations and Green's function calculations indicate an intriguing coexistence of two different Peierls-like distortions in the functionalized low-dimensional material. The presence of side defects increases the side strain and creates interband electronic states. As a result, the band gap of BNQDs could vary in a wide range depending on the type of chemical bonds and surface disorders. Enhanced edge states also improve the photoluminescence emission of the quantum dots. These side-defect enriched states in BNQDs create optical and electrical responses which could offer unprecedented potential for large scale nanophotonics such as photovoltaic, bioimaging, and quantum communication.
\end{abstract}

DOI: 10.1103/PhysRevB.96.165307

\section{INTRODUCTION}

Hexagonal boron nitride (h-BN) has been considered for unique properties such as good thermal conductivity, chemical and mechanical stability, and optical properties [1-5]. Studying the optoelectronic properties of two-dimensional (2D) $\mathrm{h}-\mathrm{BN}$, which is a wide band gap semiconductor, is currently one of the hottest topics in 2D material science. Thin BN sheets have been made by ultrasonication [2], high-energy electron beam irradiation [6], Lewis acid base [7], hydrolysis of lithium based materials [8], CVD [9], and liquid alloys of alkali metals at room temperature [10] methods. Lin et al. have shown a method to reduce the lateral size of monolayered BN to form QDs [11]. They fabricated monolayered BNQDs by exfoliating and disintegrating of hBN flakes [11]. Jung et al. [12] synthesized BNQDs by using physical energy sources including microwave irradiation, sonication, and impinging processes. They reported a defect engineering method for edge-hydroxylated functionalization to enhance the intracellular uptake of the BNQDs in cells for bioimaging [12]. Lingam et al. [13] reported that the random structure of edges of graphene quantum dots (GQDs) contributes in their photoluminescence and band gap tuning capabilities. Hassan et al. [14] experimentally studied the chemical activated graphene quantum dots by an ultrasonic approach, which could enhance the electrocapacitance and photoluminescence intensity. Side-defect BNQDs open new horizons for the design and fabrication of optoelectronics, nanophotonics, and a variety of quantum communications devices. The development of artificial atomic systems are particularly interesting for new applications in quantum technologies such as quantum

\footnotetext{
*Corresponding author: fshayeganfar@gmail.com

†Corresponding author: J.Beheshtian@srttu.edu
}

computation, communications, and metrology $[15,16]$. Recently, Tran et al. [17] demonstrated a color center in 2D $\mathrm{h}-\mathrm{BN}$ as room temperature quantum emitters, which were previously observed in silicon carbide [18], carbon nanotubes [19], nanocrystal quantum dots [20,21], and wide-band-gap semiconductors [22]. More recently, Liu et al. [23] prepared BNQDs using liquid exfoliation-solvothermal treatment of bulk BN in three different solvents. They studied their tunable blue/green photoluminescence (PL), which can be generated through different organic solvents including ethanol, $\mathrm{N}, \mathrm{N}$-dimethylformamide (DMF), and N-methyl-2-pyrrolidone (NMP) [23]. Understanding of electronic properties of $\mathrm{h}$ $\mathrm{BN}$ and its heterostructures with graphene $(\mathrm{BN} / \mathrm{G} / \mathrm{BN})$ will cause a major progress of BN-based photonic devices. These polar dielectric heterostructures at atomic scale can create nanophotonic devices as a result of hybridized optical phonons of nanocomposites [24]. Moreover, h-BN could be used as a multimode waveguide and its heterostructures with graphene could be a tunable waveguide resulting in hybridization of surface plasmon polaritons in graphene with hyperbolic phonon polaritons [24-26]. Functionalization is an important way to create peculiar electronic and optical properties and applications for a material [24]. This work presents the electronic and optical properties of functionalized BNQDs having side defect with the help of first principles density functional theory (DFT) and the Berkeley GW (BGW) package. It begins with investigation of electronic properties of the functionalized dots with different ligands in the presence of side defects. Then, the emerging optical properties and potential applications of the quantum dots are presented. Adjustable band gap of BNQDs through surface functionalization and enriched edge-electronic states would open up new opportunities for development of nanomaterials for biomedical and optoelectronic applications. We took the same size and side defect in our computational model. 


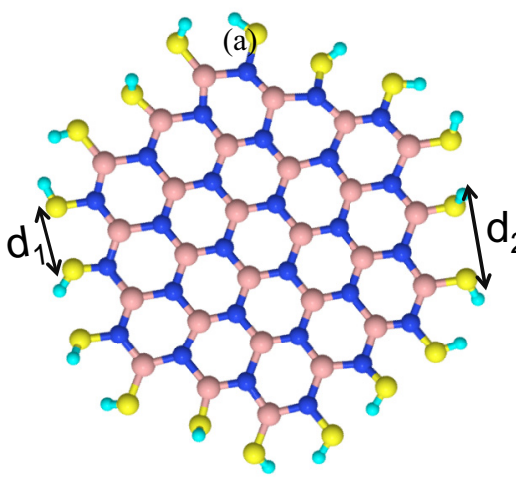

Side-defect(d)

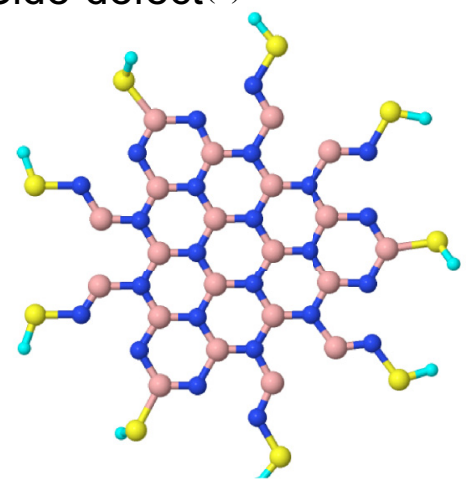

(b)

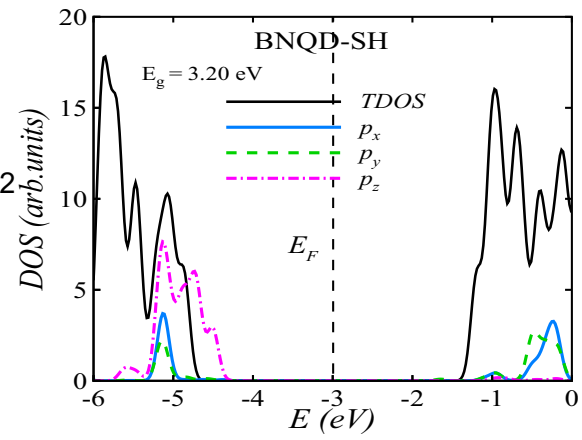

(e)

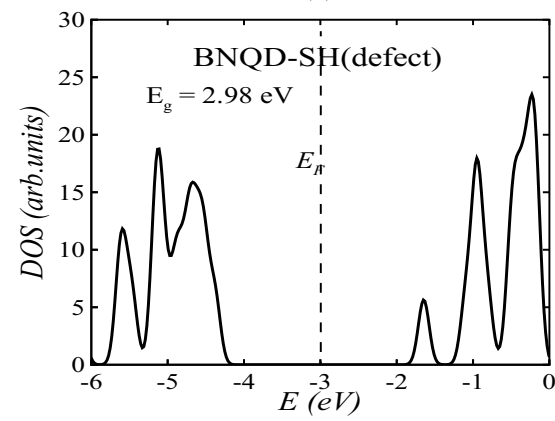

(c)

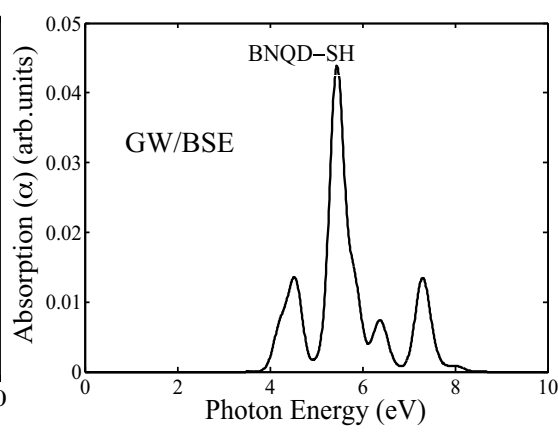

(f)

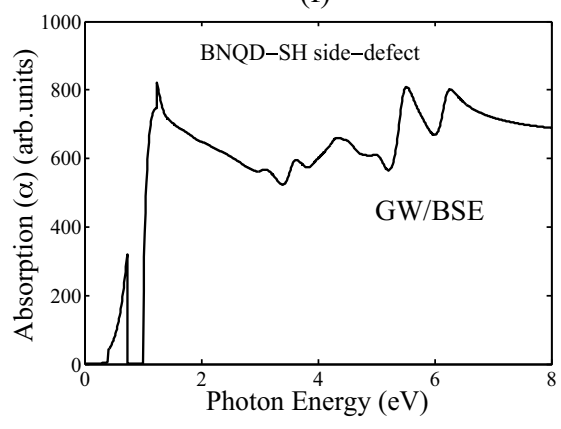

FIG. 1. (a) Relaxed geometry of BNQD-SH. (b) The total density of states (DOS) and projected DOS (PDOS) for this nanostructure. (c) The optical absorption spectrum $(\alpha)$ computed for the SH-BN. (d) Side-defect BNQD-SH. (e) The total DOS of defect structure with $E_{g}=2.98 \mathrm{eV}$. (f) The optical absorption spectrum $(\alpha)$.

\section{COMPUTATIONAL TECHNIQUE}

In this paper, we employ first-principles calculations based on density functional theory (DFT) and Green's function BGW to probe the electronic and optical properties of functionalized boron nitride quantum dots (BNQDs) and their side defect.

Electronic properties of functionalized BNQDs and their side defect were performed with the SIESTA software package based on the self-consistent density functional theory (DFT) calculations and periodic supercell method [27]. The calculations were performed within the generalized gradient approximation (GGA) in conjunction with double-zeta polarized orbital for the localized basis sets and a norm-conserving Troullier-Martins type pseudopotential for boron, nitrogen, oxygen, sulfur, and hydrogen. The convergence criteria for geometry optimizations were set to less than $0.01 \mathrm{eV} / \AA$ for forces and $10^{-5} \mathrm{eV}$ for total energy [28].

Concerning the role of dispersion force, we compare the DFT results based on the GGA and BGW-Beth-Salpeter equation (BSE) approach. Optical properties of functionalized $\mathrm{BN}$ such as hydrogen-BN (HBN), OH-BN, SH-BN, $\mathrm{NH}_{2}-\mathrm{BN}$, and $\mathrm{N}-\mathrm{BN}$ were computed using the $\mathrm{BGW}$ package based on first-principles many-body perturbation theory [29,30], with the computational details in our previous works [31,32].

In short, quasiparticle excitation energies were computed as a first-order correction to DFT within GG approximation of Perdew, Burke, and Ernzerhof, with starting DFT-PBE eigenvectors and eigenvalues taken from the SIESTA package, which is compatible with the BGW. The frequency dependence of the dielectric function is obtained via the generalized plasmonpole (GPP) model and optical excitation energies by inclusion of electron-hole interaction from the solution of BSE [30,33].

Subsequent to our GW calculations, the BSE is solved within the Tamm-Dancoff and static approximations to compute the complex transverse dielectric function, $\epsilon(\omega)=$ $\epsilon_{1}(\omega)+i \epsilon_{2}(\omega)$. The absorption coefficient, $\alpha(\omega)$, via the standard expression [34]

$$
\alpha(\omega)=2 \omega \sqrt{1 / 2\left[-\epsilon_{1}(\omega)+\sqrt{\epsilon_{1}(\omega)^{2}+\epsilon_{2}(\omega)^{2}}\right]},
$$

where $\omega$ has units of energy (in atomic units).

\section{RESULTS AND DISCUSSIONS}

\section{A. Electronic properties}

Our study begins with the investigation of electronic properties of functionalized BNQDs by $\mathrm{SH}, \mathrm{OH}, \mathrm{NH}_{2}$, and $\mathrm{N}, \mathrm{H}$ with the help of $a b$ initio quantum computation based on DFT and BGW packages as described in Sec. II. Since the optical properties of BNQDs are size dependent, $[\mathrm{BN}]_{24}$ and $[\mathrm{BN}]_{54}$ BNQDs are selected to reveal the mechanism underlying tunable electronic properties of BNQDs with different sizes. Concerning the role of size effect on the electronic and optical properties of BNQDs, we have considered two different sizes of QD by decreasing the size of the BNQD from 90 atoms to 48 atoms for functionalized BNQD, and 72 atoms to 36 atoms for side-defect structures (see Appendix for smaller supercell). We must stress that the contribution of size on the electronic 
properties generally decreases the band gap. Hence our DFT values constitute the upper values of band gap regard to larger sizes than our case study. Similar conclusions were drawn by previous reports on the dependence of electronic states quantum dots (QDs) of different shapes and sizes [35-39]. A second type of structure with side defect is also studied in order to compare with the edge-functionalized QDs. Different types of distortions due to van der Waals repulsion between close neighboring atoms create semiconductor QDs with varying optical and electronic properties. Before addressing the $\mathrm{SH}, \mathrm{OH}$, and $\mathrm{NH}_{2}$ edge-terminated QDs, we revisit the primitive $\mathrm{H}$ edgeterminated QDs (H-BN) in order to use its total density of states (DOS) as a frame of reference (for more details, see Appendix). As shown in Fig. 6, the band gap for $\mathrm{H}-\mathrm{BN}$ is $5.06 \mathrm{eV}$ with PBE (5.82 eV with GW). Table I presents the DFT energy gap, electrical dipole moment, and Peierls distortions for different edge-terminated and side defect of functionalized BNQD.

Thus we highlight the importance of side-defect edge functionalization versus perfect edge BNQDs as a route toward achieving semiconducting states. The three types of edge functionalized BNQDs are shown in Figs. 1-3. Edge functionalized BNQDs are denoted as $X-[\mathrm{BN}]_{p}-X(q)$, where $[\mathrm{BN}]_{p}$ indicates $p$ trimers of $\mathrm{BN}$ in QDs, $X$ is $\mathrm{SH}, \mathrm{OH}, \mathrm{NH}_{2}$ atoms attached to edges, and $q$ indicates the number $X$ atoms in the unit cell.

The optimized structure for the functionalized BNQDs is shown in Figs. 1(a), 2(a) and 3(a) to take into account the Peierls-like distortion and alternating tilts [40]. Figure 1(a) reveals that the trimer pairs deviate from the QD plane by out
TABLE I. DFT results for different edge-functionalized and sidedefect BNQDs, band gap $\left(E_{g}\right)$, electrical dipole moment $\vec{P}$, and distortion distance $d_{1}$ and $d_{2}$. The numbers inside parentheses are calculated by Berkeley-GW.

\begin{tabular}{lccc}
\hline \hline & \multicolumn{3}{c}{ Functionalized BNQD } \\
\cline { 2 - 4 } & $\mathrm{SH}$ & $\mathrm{OH}$ & $\mathrm{NH}_{2}$ \\
\hline$E_{\text {gap }}(\mathrm{eV})$ & $3.2(3.78)$ & $3.75(4.33)$ & $4.11(4.87)$ \\
defect & $2.98(3.45)$ & $1.14(2.06)$ & $0.96(1.77)$ \\
$|\vec{P}|($ Debye $)$ & $(0.1,0.02,0)$ & $(0.04,0,0)$ & $(1.4,10.1,0.7)$ \\
defect & $(1,0.9,0.04)$ & $(2.4,1.2,0)$ & $(1.1,13.4,3.8)$ \\
$d_{1}(\AA)$ & 2.74 & 2.65 & 2.7 \\
$d_{2}(\AA)$ & 2.86 & 2.76 & 2.81 \\
\hline
\end{tabular}

of plane distance of $0.25 \AA$ and for dimer pairs is $0.01 \AA$. The distortion distances $d_{1}(\mathrm{SN})$ and $d_{2}(\mathrm{SB})$ for $\mathrm{SH}-[\mathrm{BN}]_{54}$ $\mathrm{SH}(18)$ are elongated to 2.74 and $2.86 \AA$, respectively. The relatively larger $d_{2}$ for the dimers (SB) and the negligible tilts for the trimers (SN) imply that the dimers have a greater closedshell character than the trimers. We observed a coexistence of two different Peierls-like distortions for $X$ edge termination that manifests as a strong $X$ dimerization at the B zigzag edge and a weak $X$ trimerization at the $\mathrm{N}$ zigzag edge, dictated by the filings at the vicinity of the Fermi level. The Peierls distortion of SH-[BN] $]_{54}-\mathrm{SH}(18)$ leads to an intradimer distance of $d_{1}=$ $2.74 \AA$ for the $\mathrm{S} @ \mathrm{~N}$ and an intratrimer distance of $d_{2}=2.86 \AA$
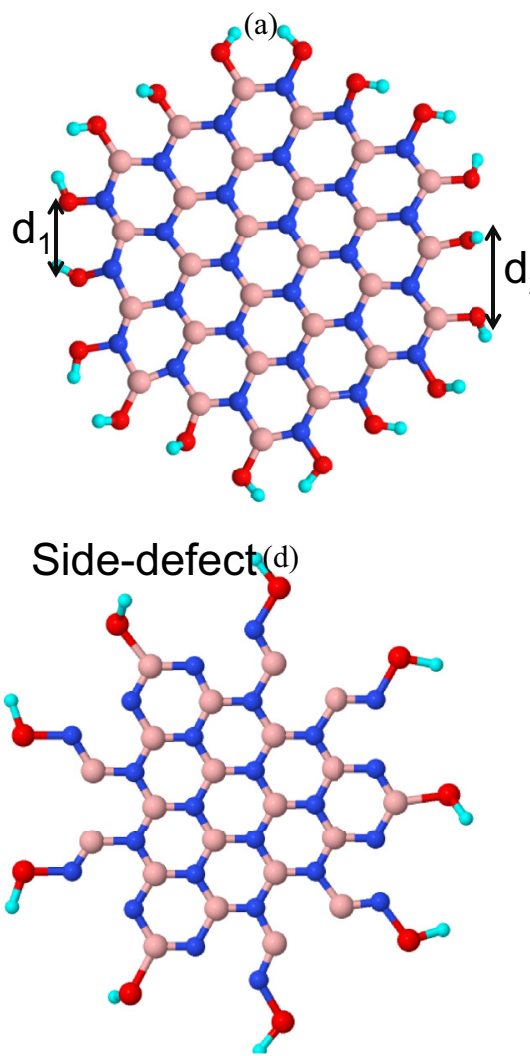

(b)

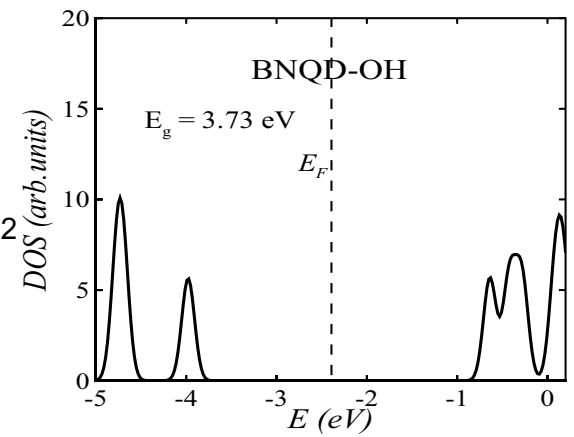

(e)

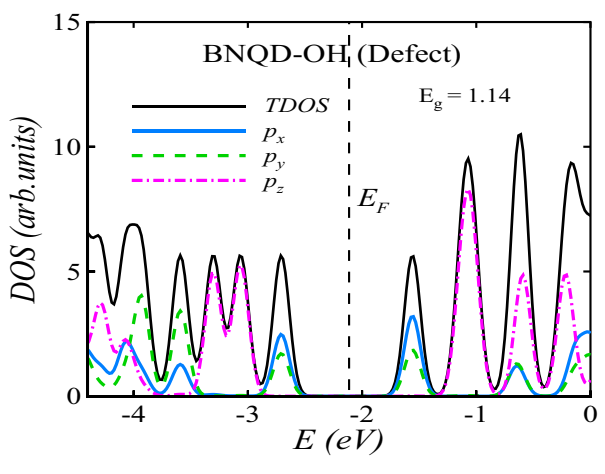

(c)

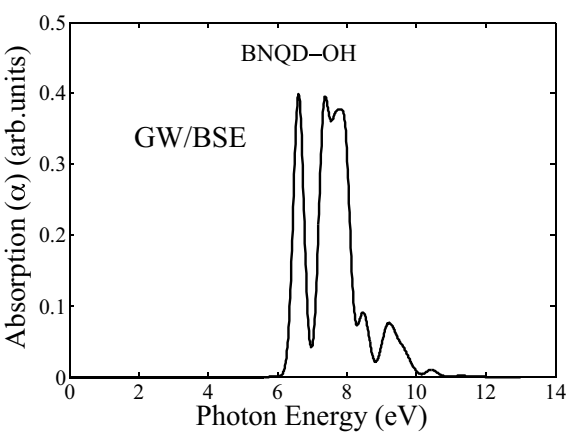

(f)

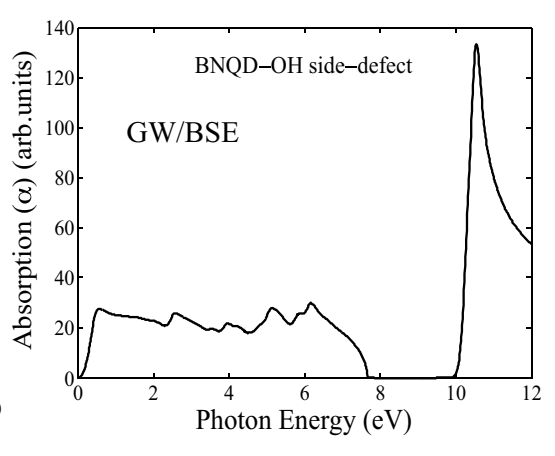

FIG. 2. (a) Relaxed geometry of BNQD-OH. (b) The total density of states (DOS) for this nanostructure. (c) The optical absorption spectrum $(\alpha)$ computed for the OH-BNQDs. (d) Side-defect BNQD-OH. (e) The total DOS and PDOS of defect structure with $E_{g}=1.14 \mathrm{eV}$. (f) The optical absorption spectrum $(\alpha)$. 

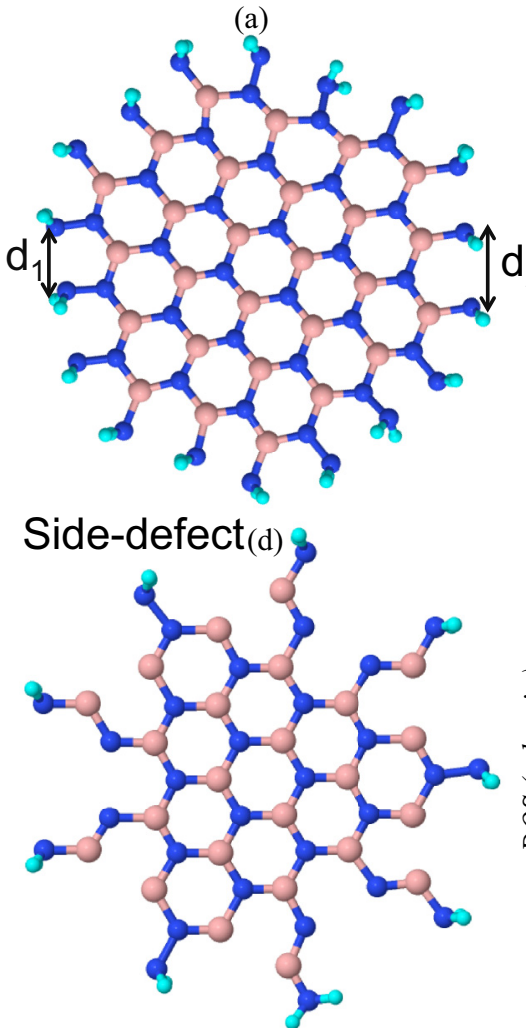

(b)

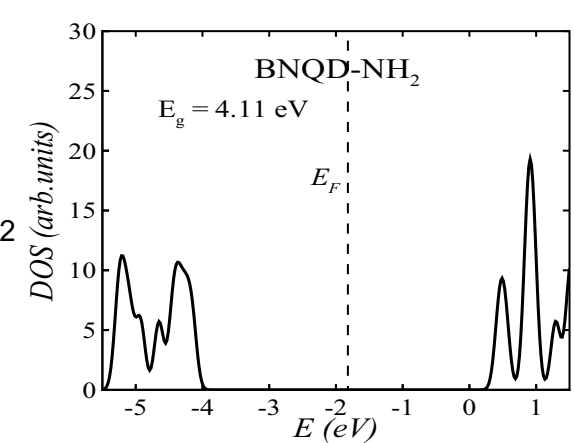

(e)

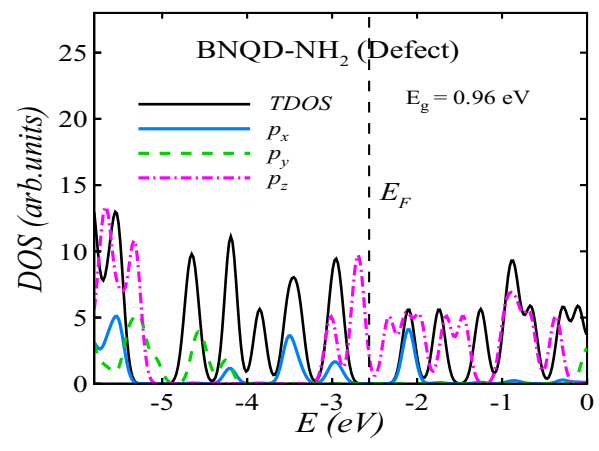

(c)

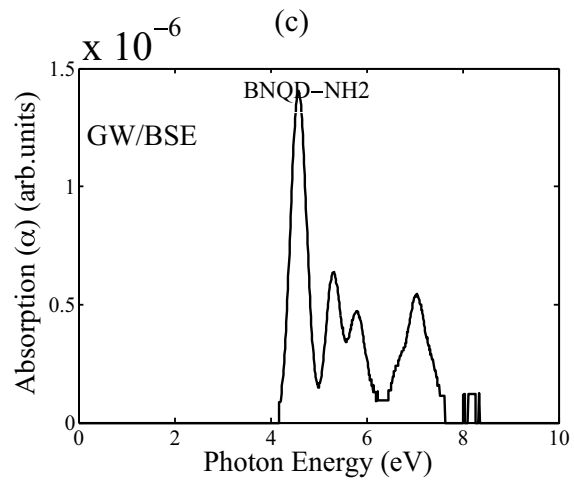

(f)

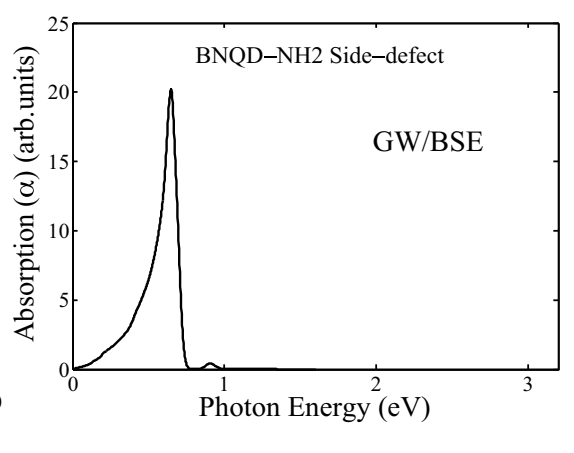

FIG. 3. (a) Relaxed geometry of BNQD-NH2 . (b) The total density of states (DOS) and projected DOS (PDOS) for this nanostructure. (c) The optical absorption spectrum $(\alpha)$ computed for the $\mathrm{NH}_{2}$-BNQDs for exciton. (d) Side-defect BNQD-NH 2 . (e) The total DOS and PDOS of defect structure with $E_{g}=0.96 \mathrm{eV}$. (f) The optical absorption spectrum $(\alpha)$.

for the $\mathrm{S} @ \mathrm{~B}$, and $\mathrm{OH}-[\mathrm{BN}]_{54}-\mathrm{OH}(18)$ intradimer distance of $d_{1}=2.65 \AA$ for the $\mathrm{O} @ \mathrm{~N}$ and intratrimer distance of $d_{2}=$ $2.76 \AA$ for the $\mathrm{O} @ \mathrm{~N}$, and $\mathrm{NH}_{2}-[\mathrm{BN}]_{54}-\mathrm{NH}_{2}(18)$ intradimer distance of $d_{1}=2.7 \AA$ for the $\mathrm{N} @ \mathrm{~N}$ and an intratrimer distance of $d_{2}=2.81 \AA$ for the $\mathrm{N} @ \mathrm{~B}$ as presented in Table I in the main manuscript. A similar Peierls-like distortion description can be done for the other functionalized QDs which brought $d_{1}, d_{2}$ distances (Table I). A similar conclusion was drawn by Lopez-Bezanilla et al. [40] for the electronic structures and magnetic properties of $\mathrm{O}$ and $\mathrm{S}$ functionalized zigzag boron

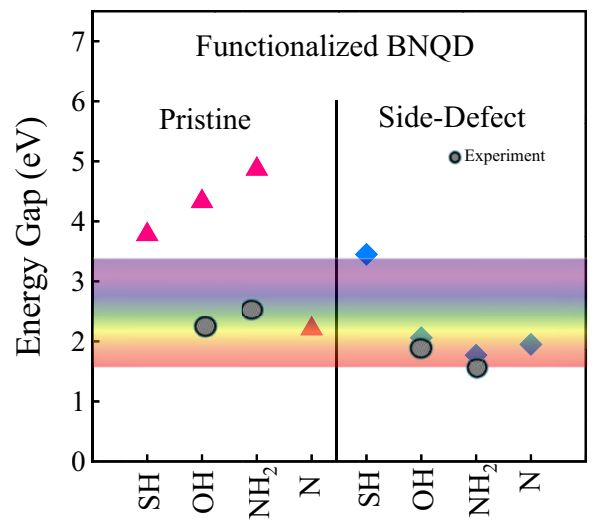

FIG. 4. Map of band gap for perfect and side-defect functionalized BNQDs, in which black circles are experimental data. Visible light range is highlighted by rainbow color in the range of $1.64-3.19 \mathrm{eV}(390-760 \mathrm{~nm})$. nitride nanoribbons (zBNNRs). Figure 1(b) shows the total density of states (DOS) and accompanying projected density of states (PDOS) of - SH atoms attached to $\mathrm{B}$ and $\mathrm{N}$ atoms. Figure 1(b) indicates that the magnitude of the band gap for SH-BNQD is $3.2 \mathrm{eV}$ with PBE (3.78 eV with $\mathrm{GW})$.

Although the primitive $\mathrm{H}$ edge-terminated QD has a large band gap $E_{g}=5.06 \mathrm{eV}$ with PBE (5.82 eV with BGW), the SH-BN exhibits semiconductor features-with an energy gap $E_{g}=3.2 \mathrm{eV}$. Comparing the PDOS of SH-[BN $]_{54}-\mathrm{SH}(36)$ with the DOS of $\mathrm{H}-\mathrm{BN}$, one may recognize that the main differences arise from the hybridization of the $S$ atoms. Each $\mathrm{S}$ atom undergoes an $s p$ hybridization yielding two hybrid orbitals, with one $s p$ forming $\mathrm{SB}$ (SN) $\sigma$ bond, whereas the other $s p$ orbital points outward to create a $\pi$ bond. A detailed analysis of projected DOS in Fig. 1(b) allows for assigning the origin of band gap closing due to the mixing of the $p_{x}, p_{y}$, and $p_{z}$ to close the gap. As a result of the dimerization, the $p_{x}$ open a gap of $4.18 \mathrm{eV}$, while trimerization for $p_{z}$ opens a gap of $3.1 \mathrm{eV}$ [Fig. 1(b)]. Note that in a dimer bond, one bonding $p_{x}$ electron is shared between one $\mathrm{S}$ center, while the two bonding $p_{x}$ electrons are shared among two $\mathrm{S}$ centers in each trimer, leading to a weaker bond and a stronger dimer bond. The weaker trimer bonds yield weaker bond length alternations in trimers than dimers, hence a smaller band gap opening at the SN edge compared to the SB edge. The electronic density of states (DOS) for the side-defect SH-BN QD is shown in Fig. 1(e). This side defect creates extra electron states (induced by strain at defect edges) causing an electrical dipole moment 
(a)

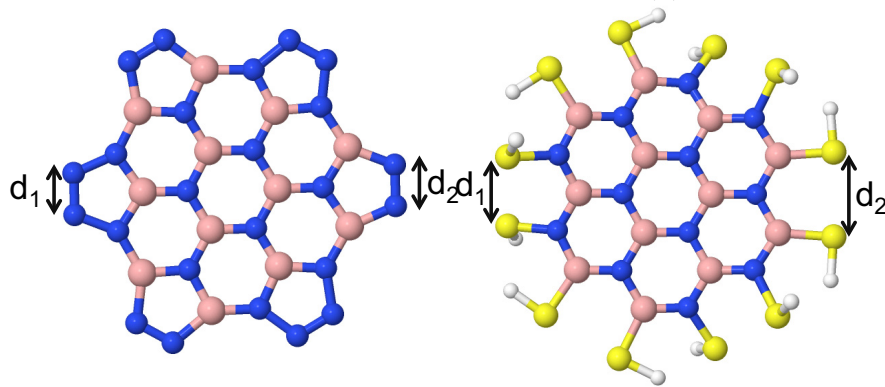

(c)

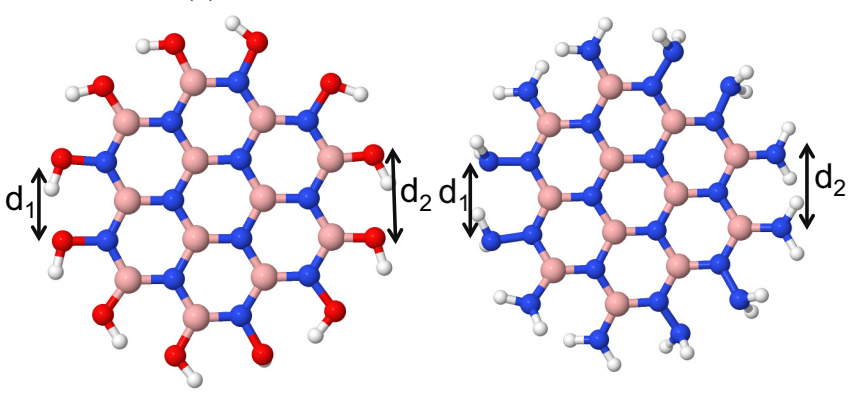

FIG. 5. Optimized functionalized BNQD, (a) N-BN, (b) SH-BN, (c) $\mathrm{OH}-\mathrm{BN}$, and (d) $\mathrm{NH}_{2}-\mathrm{BN}$.

(Table I) and band gap closing. The band gap energy for the side-defect SH-BNQD is $2.98 \mathrm{eV}$ with PBE (3.45 eV with $\mathrm{GW})$. As a conclusion, we suggest a mechanism based on the collaboration between frontier orbital hybridization and charge transfer for band gap closing consistent with previous reports [35]. Accordingly, the remarkable edge effects of BNQDs lead to more active sites and alter their electronic properties and create new phenomena. The reduced band gap originated from the edge enriched BNQDs that increased the electrical dipole moment, which could enhance intermolecular interaction and electron correlation. Figure 2(b) indicates that the magnitude of the band gap for $\mathrm{OH}-\mathrm{BNQD}$ is $3.75 \mathrm{eV}$ with PBE (4.33 eV with GW). We inspect the electronic properties of the side-defect BNQDs 2D by computing PDOS on the eigenstates of OH-BNQDs. Figure 2(e) reveals that the $p_{x}$ orbital opens a gap of $1.4 \mathrm{eV}$, while $p_{z}$ opens a gap of $2 \mathrm{eV}$ and the valence band and conduction band edge is primarily a mixture of $p_{x}$ and $p_{z}$. The projection of the contribution of $p_{z}$ orbital is greater than $p_{x}$ in the side-defect BNQDs. The resulting scenario allows us to identify a mechanism that is characterized by mixing of states of $p_{x}$ and $p_{z}$ orbitals. In simple terms, the enhanced $s p^{3}$ hybridization of the side-defect BNQDs due to enriched electronic states of edges results in the lower band gap for defect samples. Figure 3(b) indicates that the magnitude of the band gap for $\mathrm{NH}_{2}-\mathrm{BNQD}$ is $4.11 \mathrm{eV}$ with PBE (4.87 eV with $\mathrm{GW}$ ), while for the side defect of $\mathrm{NH}_{2}$-BNQD [Fig. 3(e)], the band gap is $0.96 \mathrm{eV}$ with $\mathrm{PBE}$ (1.77 with $\mathrm{GW})$.

\section{B. Optical properties}

The optical properties of BNQDs constitute a major subject of experimental and computational studies, specifically to improve BN-based optoelectronic and bioimaging devices. For instance, a photocatalytic material for using in solar cells should have wide absorption range of solar energy and band gap around $2 \mathrm{eV}$ [41]. Table I indicates that the functionalized BNQDs have a band gap of $\mathrm{SH}-\mathrm{BN}: 3.2 \mathrm{eV}$, $\mathrm{N}-\mathrm{BN}: 1.63 \mathrm{eV}, \mathrm{OH}-\mathrm{BN}: 3.75 \mathrm{eV}$, and $\mathrm{NH}_{2}: 4.11 \mathrm{eV}$, where $\mathrm{H}-\mathrm{BN}$ has a band gap of $5.06 \mathrm{eV}$. Although the electronic band gap of functionalized BNQDs is decreased, it is still too large for practical applications, which needs to be narrowed further such as creation of side-defect QDs for photocatalytic applications. To study the interaction of confined lights in nanoscale dimensions with BNQDs, we computed the absorption spectrum by using BGW packages as described in Sec. II. Figures 1(c) and 1(f) compare the optical absorption spectrum computed for SH-BN and its side defect. The SH-BN [Fig. 1(c)] shows the first peak at $5.5 \mathrm{eV}(225 \mathrm{~nm})$ and the second peak at $6.5 \mathrm{eV}(191 \mathrm{~nm})$ and $7.5 \mathrm{eV}(165 \mathrm{~nm})$. Note that all peaks are in the UV region. The optical absorption spectrum for side-defect SH-BN [Fig. 1(f)] indicates a redshift due to the smaller electronic band gap (3.2 eV). Figure 1(f) reveals that the computed lowest excitation energy for the side defect is located at $0.8 \mathrm{eV}(1550 \mathrm{~nm})$ followed by $1.15 \mathrm{eV}(1078 \mathrm{~nm})$ both in infrared, and $3.2 \mathrm{eV}(387 \mathrm{~nm})$ in visible and $3.7 \mathrm{eV}(335 \mathrm{~nm})$ in UV range. Figures 2(c) and 2(f) show the optical absorption spectrum computed for

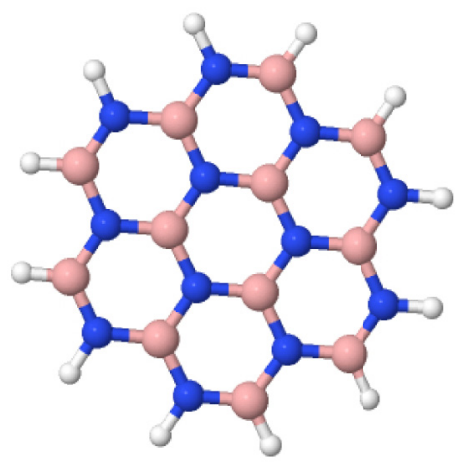

(a)

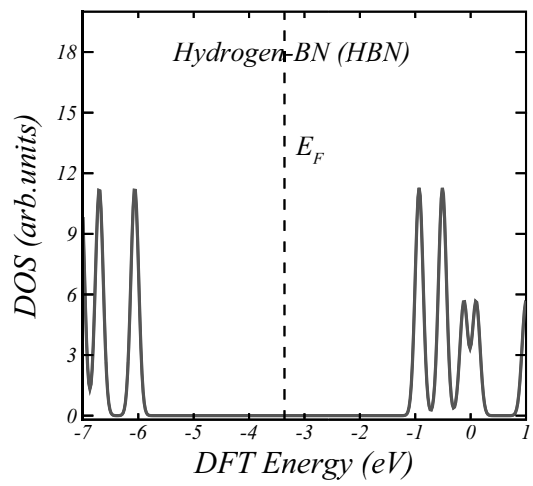

(b)

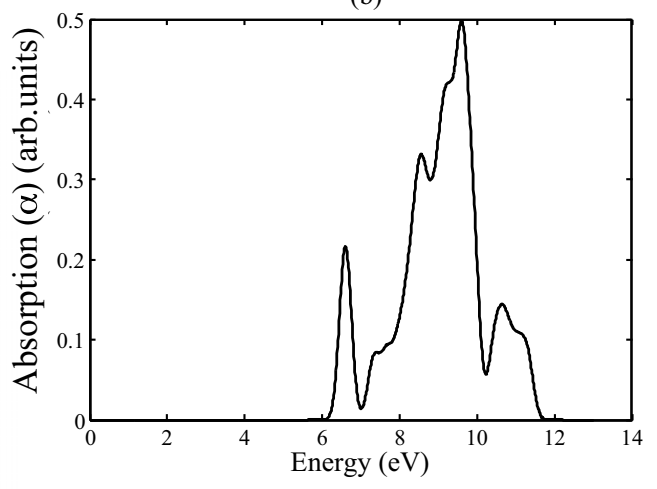

FIG. 6. Total density of states (DOS) for H edge-terminated QDs as a frame of reference, in which the band gap is $5.06 \mathrm{eV}$. (b) Absorption spectrum for H-BNQD. 
the OH-BNQDs with and without side defect. Figure 2(c) reveals that the computed lowest excitation energy for $\mathrm{OH}-\mathrm{BN}$ is located at $6.5 \mathrm{eV}$ and the several peaks in UV range. These results are in good agreement with the energy gap presented in Table I for OH-BNQDs (3.75 eV). Interestingly, side-defect $\mathrm{OH}-\mathrm{BNQDs}$ have a single emission located at $2.5 \mathrm{eV}(496 \mathrm{~nm})$. Hence more direct comparison of predicted absorption spectra for the OH-BN [Figs. 2(c) and 2(f)] with an experimental spectrum of edge-hydroxilated BN would be beneficial [12]. Jung et al. studied functionalization of BNQDs to fabricate biocompatible edge-hydroxylated BNQDs (EH-BNQDs) for bioimaging. They have found that the absorption spectrum for EH-BNQD shows (i) a peak at $320 \mathrm{~nm}$, (ii) a peak at $450 \mathrm{~nm}$ irradiated by the $280 \mathrm{~nm}$ wavelength, and (iii) a peak at $450 \mathrm{~nm}$ irradiated by the $360 \mathrm{~nm}$ wavelength [12]. Our results show that the peak maximum for the $\mathrm{NH}_{2}$-BNQDs is located at $4.5 \mathrm{eV}(275 \mathrm{~nm})$ [Figs. 3(c) and 3(f)], while with side defect this peak shifts to a longer wavelength, i.e., $1.9-2.1 \mathrm{eV}(652-590 \mathrm{~nm})$. Figure 4 summarized our findings of energy gap for perfect and side-defect functionalized BNQDs. $\mathrm{NH}_{2}$-BNQDs reveal more significant band gap closing than other chemical ligands. Moreover, we compare our theoretical results with experimental data (dark circles) in Fig. 4 for $\mathrm{NH}_{2}$ and $\mathrm{OH}-\mathrm{BNQDs}$. A close agreement exists with the experimental data.

\section{CONCLUSION}

In summary, we systematically studied the electronic and optical properties of $\mathrm{OH}, \mathrm{SH}$, and $\mathrm{NH}_{2}$ functionalized BNQDs and their side defect. We show that the functionalization on both $\mathrm{B}$ and $\mathrm{N}$ zigzag edges gives rise to band gap closing or semiconducting states and a Peierls-like distortion. Two different distortions in the form of single and double bonds coexist in the same QD. We found that functionalized BNQDs can modulate electronic and optical properties of $0 \mathrm{D}$ QDs. Moreover, their side defect acquires single quantum emission in visible light range. Activated BNQDs improve accessibility of electrolyte ions by free edges, since the edges have the ability to accumulate more charges and electron transports than the pristine BN. Our results suggest that the band gap of BNQD could be controlled by the chemical nature of functional groups and could be used to tune and control the optoelectronic properties of BNQDs. Then chemically activated BNQDs could be beneficial for applications for the next generation of miniaturized energy storage devices such as electrochemical nanocapacitors. These features suggest that the BNQDs can be good candidates to enhance photoluminescence and supercapacitance of BN-based optoelectronic device applications.

\section{ACKNOWLEDGMENTS}

The authors acknowledge use of the supercomputer machines supported in part by Rice University Research (SUR). This work was supported by Shahid Rajaee Teacher Training University.
TABLE II. DFT results for different edge-functionalized and side-defect BNQDs for $24 \mathrm{BN}$ atoms, band gap $\left(E_{g}\right)$, and distortion distance $d_{1}$ and $d_{2}$. The numbers inside parentheses are calculated by BGW.

\begin{tabular}{lccc}
\hline \hline & \multicolumn{3}{c}{ Functionalized BNQD } \\
\cline { 2 - 4 } & $\mathrm{SH}$ & $\mathrm{OH}$ & $\mathrm{NH}_{2}$ \\
\hline$E_{\text {gap }}(\mathrm{eV})$ & $3.66(4.1)$ & $4.08(4.63)$ & $4.51(5.2)$ \\
defect & $3.14(3.75)$ & $1.4(2.4)$ & $1.35(2.15)$ \\
$d_{1}(\AA)$ & 2.65 & 2.63 & 2.61 \\
$d_{2}(\AA)$ & 2.73 & 2.67 & 2.70 \\
\hline \hline
\end{tabular}

\section{APPENDIX: SIZE EFFECT ON ELECTRONIC AND OPTICAL PROPERTIES OF BNQD}

We begin with the optimized structures of functionalized BNQDs such as N-BN, SH-BN, OH-BN, and $\mathrm{NH}_{2}-\mathrm{BN}$ as shown in Fig. 5, followed by an emphasis on the electronic and optical properties of $\mathrm{H}-\mathrm{BN}$ as a reference frame and $\mathrm{N}-\mathrm{BN}$ and ends with the research perspectives.

The h-BN structures have ionicity character and a partially positively $\mathrm{B}$ and a negatively $\mathrm{N}$ charged atom. This property makes the $\mathrm{N}$ sites and the $\mathrm{B}$ sites reactive with electrophilic and nucleophilic groups, respectively. Weng et al. [24] experimentally studied numerous functional groups such as hydroxyl $(\mathrm{OH})$, amino (NH2), ether (OR), and amine (NHR), as well as heteroatoms $(\mathrm{C}$ and $\mathrm{O})$, introducing into $\mathrm{BN}$ nanostructures [24].

In order to evaluate the influence of the size of QD on electronic and optical properties of BNQD, we have also considered the different size of QD by decreasing the size of the BNQD from 90 atoms to 48 atoms for functionalized BNQD, and 72 atoms to 36 atoms for side-defect structures; such smaller QD allows us to obtain a relevant comparison of size effect on the electronic and optical properties of BNQDs. Results of smaller supercell are shown in Table II. The contribution of size for smaller unit cell on the electronic properties generally increases the band gap.

Before addressing the $\mathrm{N}, \mathrm{SH}, \mathrm{OH}$, and $\mathrm{NH}_{2}$ edgeterminated QDs, we revisit the primitive $\mathrm{H}$ edge-terminated QDs H-BN in order to use its total density of states (DOS) as a frame of reference. As shown in Fig. 6, the band gap for $\mathrm{H}-\mathrm{BN}$ is $5.06 \mathrm{eV}$ with PBE (5.82 eV with GW). Table III lists the DFT energy gap, electrical dipole moment for different $\mathrm{H}$ and $\mathrm{N}$ edge-terminated and $\mathrm{N}$ side-defect structure.

TABLE III. DFT results for $\mathrm{H}$ and $\mathrm{N}$ edge functionalized boron nitride quantum dots, energy gap $\left(E_{g}\right)$, and electrical dipole moment $\vec{P}$. The numbers inside parentheses are calculated by Berkeley-GW.

\begin{tabular}{lcc}
\hline \hline & \multicolumn{2}{c}{ Functionalized BNQD } \\
\cline { 2 - 3 } & $\mathrm{H}$ & $\mathrm{N}$ \\
\hline$E_{\text {gap }}(\mathrm{eV})$ & $5.06(5.82)$ & $1.63(2.21)$ \\
defect & & $1.2(1.95)$ \\
$|\vec{P}|($ Debye $)$ & $(1.4,10.1,0.7)$ & $(0.1,2.9,0.5)$ \\
defect & & $(8.6,13.4,0.3)$ \\
\hline \hline
\end{tabular}




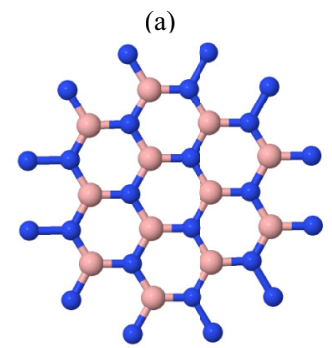

(c)

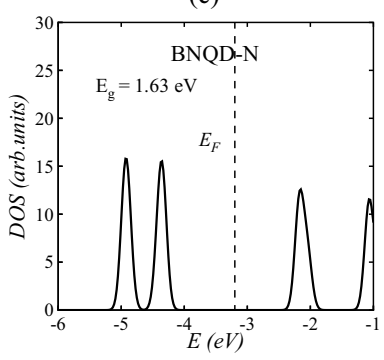

Side-defect $(\mathrm{g})$

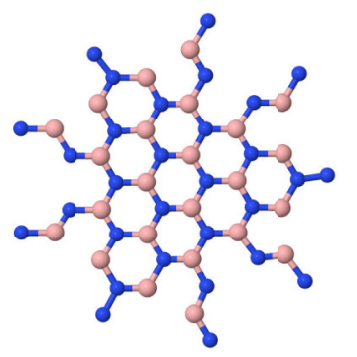

(d)

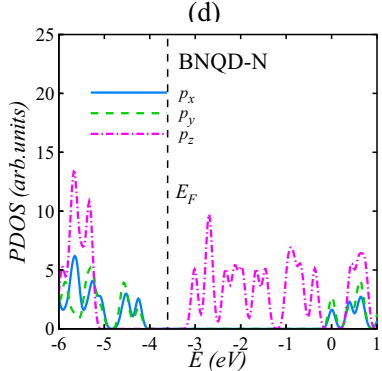

(h)

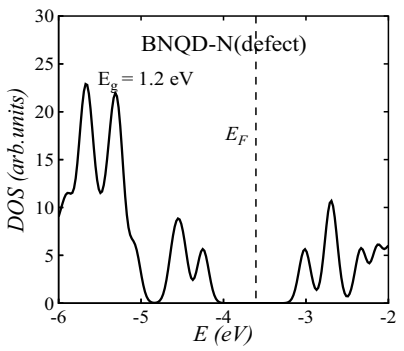

(b)

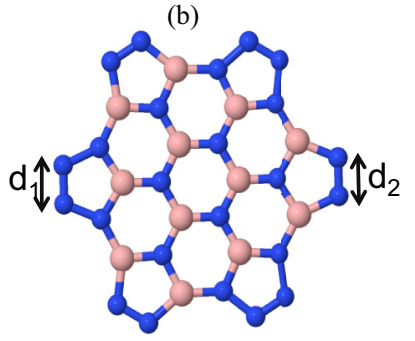

(e)

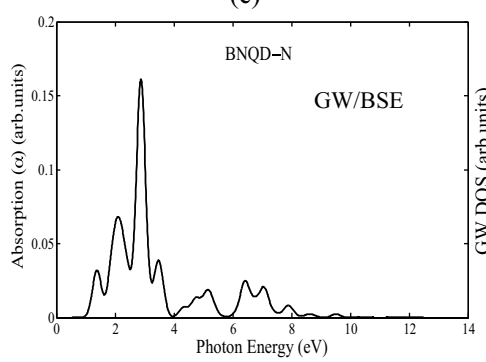

(i)

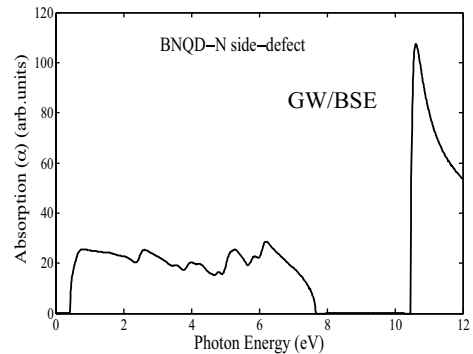

(f)

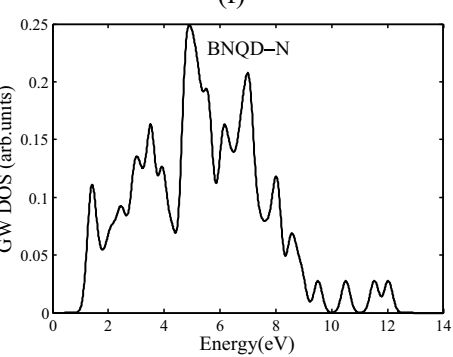

(j)

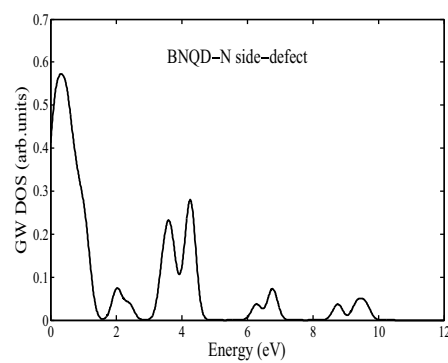

FIG. 7. (a) Geometry of BNQD-N and (b) fully relaxed geometry. (c) The total density of states (DOS) for this nanostructure with $E_{g}=1.63 \mathrm{eV}$ and (d) projected DOS (PDOS). (e) The optical absorption spectrum $(\alpha)$ computed for exciton BNQD-N, showing three distinct peaks in the visible region $(\sim 1.6-3.2 \mathrm{eV}$ ). (f) GW-DOS of exciton BNQD-N, which is computed by Berkeley-GW. (g) Side-defect BNQD-N. (h) Total DOS of defect structure, with $E_{g}=1.2 \mathrm{eV}$. (i),(j) The optical absorption coefficient $(\alpha)$ and GW-DOS for defect structure, respectively.

The geometry of BNQD-N and fully relaxed geometry are plotted in Figs. 7(a) and 7(b). Figures 7(c) and 7(d) show the total density of states (DOS) for N-BNQD and projected DOS (PDOS), the energy gap of which is $E_{g}=1.63 \mathrm{eV}$. The optical absorption spectrum $(\alpha)$ computed for the N-BN quantum dots (QD) for exciton [Fig. 7(e)], showing three distinct peaks in the absorption spectrum, was computed by Berkeley-GW for BNQD-N.

The electronic density of states for the side-defect N-BN QD is shown in Fig. 7(h). This side defect creates extra electron states, causing the electrical dipole moment (Table III) and band gap closing. The magnitude of the band gap for sidedefect N-BNQD is $1.2 \mathrm{eV}$ with PBE (1.95 eV with GW). Accordingly, the remarkable edge effects of BNQDs lead to more active sites and alter their electronic properties and create different phenomena as discussed in the main manuscript.

To study the interaction of confined lights in nanoscale dimensions with BNQD, we computed absorption spectrum by using BGW packages. In Figs. 7(e) and 7(i) for N-BNQD, several peaks in the visible region exist. The first peak in the absorption spectrum is located at $1.65 \mathrm{eV}(751 \mathrm{~nm})$, followed by $2.1 \mathrm{eV}(590 \mathrm{~nm})$ and $3.0 \mathrm{eV}(413 \mathrm{~nm})$. Accordingly, for side-defect N-BN, a wide spectrum exists in the visible light region.

Our DFT results on side-defect functionalized BNQDs suggest that the band gap closing depends on the chemical functional groups, modulating optoelectronic properties of BNQDs. Peculiar properties of functionalized BNQDs allow for band gap engineering as a major requirement of various QD devices.
[1] K. Watanabe, T. Taniguchi, and H. Kanda, Nat. Mater. 3, 404 (2004).

[2] C. Zhi, Y. Bando, C. Tang, H. Kuwahara, and D. Golberg, Adv. Mater. 21, 2889 (2009).

[3] L. H. Li, J. Cervenka, K. Watanabe, T. Taniguchi, and Y. Chen, ACS Nano 8, 1457 (2014).
[4] Y. Lin and J. W. Connell, Nanoscale 4, 6908 (2012).

[5] P. Sutter, J. Lahiri, P. Zahl, B. Wang, and E. Sutter, Nano Lett. 13, 276 (2012).

[6] C. Jin, F. Lin, K. Suenaga, and S. Iijima, Phys. Rev. Lett. 102, 195505 (2009).

[7] L. Cao, S. Emami, and K. Lafdi, Mater. Express 4, 165 (2014). 
[8] J. Zhu, H. Wang, J. Liu, L. Ouyang, and M. Zhu, Nanotechnology 28, 115604 (2017).

[9] L. Song, L. Ci, H. Lu, P. B. Sorokin, C. Jin, J. Ni, A. G. Kvashnin, D. G. Kvashnin, J. Lou, B. I. Yakobson, and P. M. Ajayan, Nano Lett. 10, 3209 (2010).

[10] H. Feng, Z. Hua, and X. Liu, Chem. Commun. 51, 10961 (2015).

[11] L. Lin, Y. Xu, S. Zhang, I. M. Ross, A. C. M. Ong, and D. A. Allwood, Small 10, 60 (2014).

[12] J.-H. Jung, M. Kotal, M.-H. Jang, J. Lee, Y.-H. Cho, W.-J. Kim and I.-K. Oh, RSC Adv. 6, 73939 (2016).

[13] K. Lingam, R. Podila, H. Qian, S. Serkiz, and A. M. Rao, Adv. Funct. Mater. 23, 5062 (2013).

[14] M. Hassan, E. Haque, K. R. Reddy, A. I. Minett, J. Chenc, and V. G. Gomes, Nanoscale 6, 11988 (2014).

[15] J. L. Brien, A. Furusawa, and J. Vuckovic, Nat. Photon. 3, 687 (2009).

[16] D. D. Awschalom, L. C. Bassett, A. S. Dzurak, E. L. Hu, and J. R. Petta, Science 339, 1174 (2013).

[17] T. T. Tran, K. Bray, M. J. Ford, M. Toth, and I. Aharonovich, Nat. Nanotechnol. 11, 37 (2016).

[18] S. Castelletto, B. C. Johnson, V. Ivady, N. Stavrias, T. Umeda, A. Gali, and T. Ohshima, Nat. Mater. 13, 151 (2014).

[19] X. Ma, N. F. Hartmann, J. K. Baldwin, S. K. Doorn, and H. Htoon, Nat. Nanotechnol. 10, 671 (2015).

[20] B. D. Mangum, F. Wang, A. M. Dennis, Y. Gao, X. Ma, J. A. Hollingsworth, and H. Htoon, Small 10, 2892 (2014).

[21] P. Michler, A. Imamoğlu, M. D. Mason, P. J. Carson, G. F. Strouse, and S. K. Buratto, Nature (London) 406, 968 (2000).

[22] I. Aharonovich and E. Neu, Adv. Opt. Mater. 2, 911 (2014).

[23] M. Liu, Y. Xu, Y. Wang, X. Chen, X. Ji, F. Niu, Z. Song, and J. Liu, Adv. Opt. Mater. 5, 1600661 (2017).

[24] Q. Weng, X. Wang, X. Wang, Y. Bandoa, and D. Golberg, Chem. Soc. Rev. 45, 3989 (2016).

[25] S. Dai, Q. Ma, M. Liu, T. Andersen, Z. Fei, M. Goldflam, M. Wagner, K. Watanabe, T. Taniguchi, M. Thiemens, F. Keilmann,
G. C. A. M. Janssen, S.-E. Zhu, P. Jarillo-Herrero, M. M. Fogler, and D. N. Basov, Nat. Nanotechnol. 10, 682 (2015).

[26] L. Ju, J. Velasco, Jr., E. Huang, S. Kahn, C. Nosiglia, H.-Z. Tsai, W. Yang, T. Taniguchi, K. Watanabe, Y. Zhang, G. Zhang, M. Crommie, A. Zettl, and F. Wang, Nat. Nanotechnol. 9, 348 (2014).

[27] J. M. Soler, E. Artacho, J. D. Gale, A. Garca, J. Junquera, P. Ordejon, and D. Sanchez-Portal, J. Phys.: Condens. Matter 14, 2745 (2002).

[28] F. Shayeganfar, K. S. Vasu, R. R. Nair, F. M. Peeters, and M. Neek-Amal, Phys. Rev. B 95, 144109 (2017).

[29] S. Sharifzadeh, A. Biller, L. Kronik, and J. B. Neaton, Phys. Rev. B 85, 125307 (2012).

[30] J. Deslippe, G. Samsonidze, D. A. Strubbe, M. Jain, M. L. Cohen, and S. G. Louie, Comput. Phys. Commun. 183, 1269 (2012).

[31] F. Shayeganfar, J. Beheshtiyan, M. Neek-Amal, and R. Shahsavari, Nanoscale 9, 4205 (2017).

[32] S. Sharifzadeh, P. Darancet, L. Kronik, and J. B. Neaton, J. Phys. Chem. Lett. 4, 2197 (2013).

[33] M. S. Hybertsen and S. G. Louie, Phys. Rev. B 34, 5390 (1986).

[34] G. Grosso and G. Parravicini, Solid State Physics, 2nd ed. (Academic Press, London, 2003).

[35] Y. Li, H. Shu, X. Niu, and J. Wang, J. Phys. Chem. C 119, 24950 (2015).

[36] L. Liang and W. Xie, Physica B 462, 15 (2015).

[37] S. Gaan, G. He, and R. M. Feenstra, J. Appl. Phys. 108, 114315 (2010).

[38] C. Y. Ngo, S. F. Yoon, W. K. Loke, Q. Cao, D. R. Lim, V. Wong, Y. K. Sim, and S. J. Chua, Nanoscale Res. Lett. 3, 486 (2008).

[39] J. Feng, H. Dong, L. Yu, and L. Dong, J. Mater. Chem. C 5, 5984 (2017).

[40] A. Lopez-Bezanilla, J. Huang, H. Terrones, and B. G. Sumpter, Nano Lett. 11, 3267 (2011).

[41] H. Zhang, C.-J. Tong, Y. Zhang, Y.-N. Zhang, and L.-M. Liu, J. Mater. Chem. A 3, 9632 (2015). 